\author{
Piero LEO* - Leonardo FORBICIONI**
}

\title{
Nuovi dati faunistici su Tenebrionidi dell'Arcipelago Toscano (Coleoptera Tenebrionidae)
}

\begin{abstract}
Riassunto: Vengono forniti nuovi dati sulla distribuzione nell'Arcipelago Toscano di 19 specie di Tenebrionidae. Dichillus corsicus (Solier, 1838) risulta nuovo per l'isola di Montecristo, l'Arcipelago Toscano e la regione Toscana; Eledona agricola (Herbst, 1783), Palorus depressus (Fabricius, 1790), Uloma culinaris (Linnaeus, 1758), Diaperis boleti (Linnaeus, 1758), Pentaphyllus chrysomeloides (Rossi, 1792), Corticeus pini (Panzer, 1799) e Scaphidema metallica (Fabricius, 1792) sono segnalati per la prima volta per l'Isola d'Elba e l'Arcipelago Toscano; Phaleria acuminata acuminata Küster, 1852 viene segnalata per la prima volta per Pianosa e Arcipelago Toscano; Dichillus minutus (Solier, 1838) viene segnalato per la prima volta per Cerboli, Dichillus tyrrhenicus Leo, 2008 e Gonocephalum obscurum obscurum (Küster, 1849) per Pianosa, Alphitobius diaperinus (Panzer, 1797) per l'Elba, Catomus rotundicollis (Guérin-Méneville, 1825) per Cerboli e Gorgona, Colpotus strigosus strigosus (A. Costa, 1847) per il Giglio, Crypticus gibbulus (Quensel, 1806) e Phaleria bimaculata bimaculata (Linnaeus, 1767) per Montecristo; vengono inoltre resi noti i primi reperti di tre specie di Tenebrionidae per l'isolotto di Palmaiola: Nalassus planipennis (Küster, 1850), Dendarus coarcticollis (Mulsant, 1854) e Colpotus strigosus strigosus. Le tribù Bolitophagini, Ulomini (sottofamiglia Tenebrioninae), Hypophlaeini e Scaphidemini (sottofamiglia Diaperinae) sono anch'esse nuove per l'Arcipelago Toscano. Viene anche illustrato l'habitus delle tre specie del genere Dichillus Jaquelin Du Val, 1861 presenti nell'arcipelago.
\end{abstract}

\begin{abstract}
New faunistic data about tenebrionid beetles of the Tuscan Archipelago (Coleoptera Tenebrionidae).
New data are provided on the distribution in the Tuscan Archipelago of 19 species of Tenebrionidae. Dichillus corsicus (Solier, 1838) is new for Montecristo, the Tuscan Archipelago and the region of Tuscany; Eledona agricola (Herbst, 1783), Palorus depressus (Fabricius, 1790), Uloma culinaris (Linnaeus, 1758), Diaperis boleti (Linnaeus, 1758), Pentaphyllus chrysomeloides (Rossi, 1792), Corticeus pini (Panzer, 1799) and Scaphidema metallica (Fabricius, 1792) are reported for the first time for the Island of Elba and the Tuscan Archipelago; Phaleria acuminata acuminata Küster, 1852 is reported for the first time for Pianosa and the Tuscan Archipelago; Dichillus minutus (Solier, 1838) is reported for the first time for Cerboli, Dichillus tyrrhenicus Leo, 2008 and Gonocephalum obscurum obscurum (Küster, 1849) for Pianosa, Alphitobius diaperinus (Panzer, 1797) for Elba, Catomus rotundicollis (Guérin-Méneville, 1825) for Cerboli and Gorgona, Colpotus strigosus strigosus (A. Costa, 1847) for Giglio, Crypticus gibbulus (Quensel, 1806) and Phaleria bimaculata bimaculata (Linnaeus, 1767) for Montecristo; the first records of three species of Tenebrionidae for the islet of Palmaiola are reported: Nalassus planipennis (Küster, 1850), Dendarus coarcticollis (Mulsant, 1854) and Colpotus strigosus strigosus. The tribes Bolitophagini, Ulomini (subfamily Tenebrioninae), Hypophlaeini and Scaphidemini (subfamily Diaperinae) are new for the Tuscan Archipelago. The habitus of the three species of the genus Dichillus Jaquelin Du Val, 1861 inhabiting the archipelago, is also illustrated.
\end{abstract}

Key words: Coleoptera, Tenebrionidae, new records, Tuscan Archipelago, Italy.

\section{INTRODUZIONE}

Le moderne conoscenze sui Tenebrionidi delle isole toscane derivano anzitutto dall'approfondito studio di Gardini (1976) che, per la prima volta, prende in esame l'insieme delle specie di questa famiglia raccolte nell'intero arcipelago, revisionando criticamente le precedenti citazioni e studiando una gran mole di materiali raccolti in epoche diverse da vari studiosi. Alcuni contributi successivi hanno aggiunto altri dati faunistici e sistematici (Gardini, 1979; Leo, 1998, 2008, 2015; Fattorini \& Leo, 2000; Lo Cascio et al., 2000), mentre considerazioni zoogeografiche vengono proposte da Fattorini (2009). Nel presente lavoro sono resi noti nuovi reperti, che ampliano in modo sensibile il numero di taxa conosciuti per l'intero arcipelago e per diverse isole dello stesso. Si è anche ritenuto opportuno fornire una foto dell'habitus dei Dichillus Jaquelin Du Val, 1861 presenti nelle isole toscane, come contributo alla conoscenza di questo genere che, in passato, ha spesso portato a discordanza nell'interpretazione e nel riconoscimento delle specie.

\section{MATERIALI E METODI}

La gran parte dei reperti è stata effettuata da

\footnotetext{
*Piero Leo, Via Tola 21, 09128 Cagliari, Italia. E-mail: piero.leo@tiscali.it

${ }^{* *}$ Leonardo Forbicioni, World Biodiversity Association onlus c/o NAT LAB Forte Inglese, 57037 Portoferraio (LI), Italia. E-mail: arcipelago.toscano@biodiversityassociation.org
} 
uno degli autori (L.F.), che, risiedendo con continuità a Portoferraio, ha potuto svolgere negli ultimi 10 anni ricerche faunistiche approfondite sulle varie isole dell'arcipelago. Gli esemplari sono stati raccolti nel corso di una serie di programmi di ricerca autorizzate dal P.N.A.T. (Parco Nazionale dell'Arcipelago Toscano) e in parte promosse dalla W.B.A. (World Biodiversity Association Onlus) di Verona, finalizzati allo studio e al censimento dell'entomofauna delle isole dell'Arcipelago Toscano. Le ricerche sono state effettuate con i metodi comunemente utilizzati per le raccolte entomologiche, ovvero, con l'utilizzo dell'ombrello entomologico, retino da sfalcio, ispezione diretta sotto le cortecce e sotto pietre, nonché vagliando detriti vegetali e lettiera. Solo raramente sono state utilizzate trappole a caduta contenenti aceto e cloruro di sodio e, in alcuni casi, la ricerca diretta "a vista", in orario notturno, sui muri $o$ in prossimità di manufatti. Nel corso di questi ultimi 10 anni, le ricerche hanno riguardato tutte le isole, e gli isolotti, nella maniera più omogenea possibile, seppure condizionate dalle problematiche logistiche riscontrate per l'accesso ad alcune di esse (es. Montecristo e Gorgona) durante le stagioni meno favorevoli. I materiali sono conservati nelle raccolte personali degli autori.

Le foto sono state eseguite da uno degli autori (L.F.) con fotocamera Canon EOS 600D e obiettivo Canon Macro f/2.8 $100 \mathrm{~mm}$; gli scatti, a diversi livelli di fuoco, sono stati combinati con la tecnica del "stacking-focus" utilizzando il software Zerene Stacker.

Le specie sono elencate seguendo l'ordine e la nomenclatura di Löbl et al. (2008). Le località dei reperti sono riportate in ordine alfabetico per isola, eventuale comune (limitatamente all'isola d'Elba) ed eventuale toponimo più ristretto. Le sigle delle province non sono indicate, tenendo presente che, tra le isole citate, Capraia, Cerboli, Elba, Gorgona, Montecristo, Palmaiola e Pianosa appartengono alla provincia di Livorno, mentre Giglio fa capo alla provincia di Grosseto.

\author{
Abbreviazioni \\ CLF: Collezione L. Forbicioni (Portoferraio) \\ CPL: Collezione P. Leo (Cagliari) \\ LF: L. Forbicioni legit \\ ex: esemplare/i \\ rs: resto
}

\section{ELENCO DELLE SPECIE}

Dichillus (Dichillus) corsicus (Solier, 1838) Fig. 1 RePERTI: Isola di Montecristo, Cala Maestra, 78.VI.2015, LF, 5 ex (CLF, CPL); idem, idem, 11.X.2015, LF, 2 ex (CLF); idem, Collo dei Lecci, 30.IX.2016, LF, 2 ex (CLF, CPL); idem, idem, 3.X.3016, LF, 8 ex (CLF, CPL).

Note. Specie tirrenica, ampiamente diffusa in Sardegna e Corsica, con alcune stazioni nell'Italia meridionale: Campania, Basilicata e Calabria (Leo, 2008). Le precedenti citazioni per l'Arcipelago Toscano (Lo Cascio, 2001; Aliquò et al., 2007; Fattorini, 2009) sono dovute a confusione con Dichillus tyrrhenicus Leo, 2008 (cfr. Leo, 2008: 608-609). Prima segnalazione per Montecristo, per l'Arcipelago Toscano e per la regione Toscana.



Fig. 1. Dichillus (Dichillus) corsicus (Solier, 1838) di Isola Montecristo, Cala Maestra (3,5 mm). 
Dichillus (Dichillus) minutus (Solier, 1838) Fig. 2 REPERTI: Isolotto di Cerboli, 28.VIII.2015, LF, 1 ex (CLF).

Note. Specie nota di Catalogna, Francia meridionale e gran parte dell'Italia peninsulare (Leo, 2015); già segnalata per l'Arcipelago Toscano di Capraia, Monte Argentario (Gardini, 1976) e dell'Elba (Leo, 2015). Prima segnalazione per l'Isolotto di Cerboli.

Dichillus (Dichillus) tyrrhenicus Leo, 2008 Fig. 3 REPERTI: Isola di Pianosa, campo sportivo, 14.II.2014, LF, 1 ex (CLF).

Note. Specie nota di Sardegna, Corsica e Arcipelago Toscano, che in passato è stata male interpretata e confusa con altri taxa (Leo, 2008). Citata da Gardini (1976) per l'Elba, il Giglio, Capraia e Montecristo, con il nome di "Dichillus (s. str.) pumilus (Solier)";

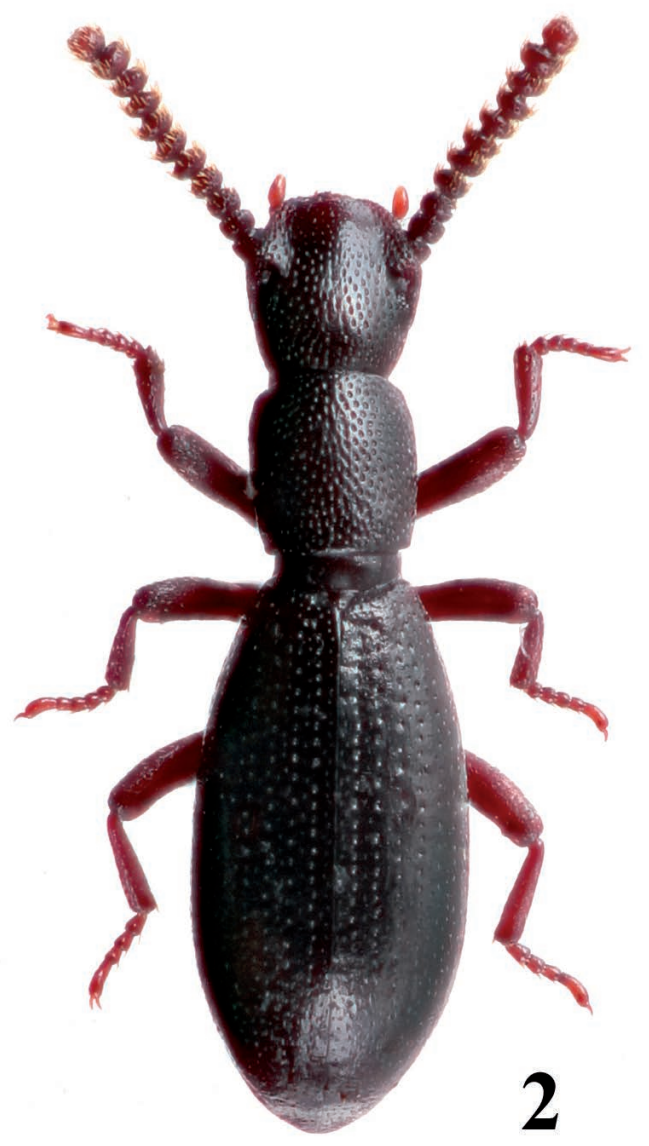

Fig. 2. Dichillus (Dichillus) minutus (Solier, 1838) di Isola d'Elba, Marciana, M.te Capanne (3,2 mm). indicata per le stesse isole da Lo Cascio (2001) sub "Dichillus corsicus (Solier, 1838)" e da Fattorini (2009) sub "Dichillus corsicus (Solier, 1838) complex". Segnalata anche dell' isola fossile di Monte Argentario (Leo, 2008), unica località peninsulare nota per la specie. Prima segnalazione per Pianosa.

\section{Alphitobius diaperinus (Panzer, 1797)}

RePerTI: Isola d'Elba, Porto Ferraio, loc. Albereto, 2.X.2010, G. Frangini leg., 1 ex (CLF).

Note. Specie subcosmopolita, legata ad attività antropiche come gli allevamenti avicunicoli e lo stoccaggio di derrate alimentari e mangimi. Diffusa in tutte le regioni italiane (Aliquò et al., 2007), era nota per l'Arcipelago Toscano solo dell'Isola del Giglio, per reperti di G. Doria risalenti agli anni 1900-1901 (cfr. Gardini, 1976). Prima segnalazione per l'Elba.



Fig. 3. Dichillus (Dichillus) tyrrhenicus Leo, 2008 di Isola d'Elba, Capoliveri, M.te Calamita (3,3 mm). 
Eledona agricola (Herbst, 1783)

REPERTI: Isola d'Elba, Marciana, M. Capanne, cabinovia, 700 m, 29.VII.2012, LF, 10 ex (CLF, CPL); idem, Portoferraio, dintorni Monte Fabrello, 8.V.2013, LF, 2 ex (CLF).

Note. Specie ad ampia distribuzione europea (Löbl et al., 2008), citata genericamente di tutta Italia da Luigioni (1929) e Porta (1934); tuttavia Aliquò et al. (2007) non la indicano in mappa per varie regioni italiane, tra cui la Toscana. Prima segnalazione (sia della specie che della tribù Bolitophagini) per l'Elba e per l'Arcipelago Toscano; primo reperto dettagliato per la regione Toscana.

Nalassus (Nalassus) planipennis (Küster, 1850) REPERTI: Isolotto Palmaiola, 28.VIII.2015, LF, 1 rs (CLF).

NotE. Specie endemica dell'Italia peninsulare, poco frequente, diffusa soprattutto nelle regioni tirreniche (Gardini, 1976). Per l'Arcipelago Toscano era già nota dell'Elba e dell'isola fossile di Monte Argentario (Gardini, 1976). Prima segnalazione per l'Isolotto di Palmaiola.

Catomus (Catomus) rotundicollis (Guérin-Méneville, 1825)

REPERTI: Isolotto di Cerboli, 28.VIII.2015, LF, 1 rs (CLF). Isola Gorgona, sopra centro abitato, 8.V.2015, LF, 1 ex (CLF).

Note. Specie mediterraneo-occidentale, ampiamente diffusa nelle regioni italiane (cfr. Aliquò et al., 2007) e soprattutto frequente sul versante tirrenico. Precedentemente segnalata per 1'Arcipelago Toscano di Elba, Giglio, Capraia, Giannutri, Pianosa e Isolotto La Scola (Gardini, 1976, 1979; Lo Cascio et al., 2000). Prime segnalazioni per Gorgona e Cerboli.

\section{Gonocephalum (Gonocephalum) obscurum obscu- rum (Küster, 1849)}

REPERTI: Isola di Pianosa, dint. paese, 14.II.2014, LF, 4 ex (CLF, CPL).

Note. Specie afrotropicale-mediterranea (Iwan et al., 2010), presente in Italia nelle regioni tirreniche e in Puglia (Aliquò et al., 2007). Per l'Arcipelago Toscano è già stata citata da Gardini (1976) di Elba e Capraia, sub "Gonocephalum (s. str.) prolixum ssp. prolixum (Erichson)". Prima segnalazione per Pianosa.

Palorus depressus (Fabricius, 1790)

REPERTI: Isola d'Elba, Campo nell'Elba, Bonalaccia,
6.III.2011, sub cort. Eucalyptus, LF, 9 ex (CLF); idem, Portoferraio, S. Giovanni, Terme, 2.II.2013, LF, 1 ex (CLF); idem, Portoferraio, Acquabona, 15.I.2011, LF, 2 ex (CPL); idem, idem, 30.XII.2012, LF, 1 ex (CLF); idem, Portoferraio, Buraccio, 21.V.2013, LF, 1 ex (CLF); ); idem, Portoferraio, Volterraio, 25.IV.2013, LF, 1 ex (CLF); idem, Marciana, Monte Capanne, sentiero per S. Cerbone, 1.II.2013, LF, 1 ex (CLF).

Note. Specie ad ampia distribuzione europea (Löbl et al., 2008), citata genericamente di tutta Italia da Luigioni (1929) e Porta (1934); tuttavia Aliquò et al. (2007) non la indicano in mappa di tutte le regioni dell'Italia centrale; più recentemente son state riportate segnalazioni dettagliate per Campania (Fattorini, 2007), Lazio (Fattorini, 2013) e Toscana (Papi \& Ceccolini, 2014). Prima segnalazione per l'Elba e per l'Arcipelago Toscano.

Dendarus (Dendarus) coarcticollis (Mulsant, 1854) REPERTI: Isolotto Palmaiola, 28.VIII.2015, LF, 1 rs (CLF).

Note. Specie presente in Austria, Svizzera, Francia, Italia e Croazia (Löbl et al., 2008); in Italia è ampiamente diffusa nelle regioni settentrionali e centrali (Papi \& Ceccolini, 2014). Già citata per l'Arcipelago Toscano di Elba, Giglio, Capraia, Montecristo, Gorgona, Pianosa e Giannutri (Gardini, 1976, sub "Dendarus tristis Laporte de Castelnau"). Prima segnalazione per l'Isolotto di Palmaiola.

\section{Colpotus strigosus strigosus (A. Costa, 1847)}

RePERTI: Isola del Giglio, Giglio Castello, c/o sorgente, $385 \mathrm{~m}, \quad 42^{\circ} 21^{\prime} 17.9^{\prime \prime} \mathrm{N} \quad 10^{\circ} 54^{\prime} 09.4^{\prime \prime} \mathrm{E}$, 9.IV.2016, Bellò, Chemello \& Forbicioni legg., vaglio Hedera in lecceta, 1 ex (CLF). Isolotto Palmaiola, 28.VIII.2015, LF, 1 ex (CLF).

Note. Specie endemica italiana, politipica, diffusa nelle regioni peninsulari e in Sicilia (cfr. Gardini, 1976; Aliquò \& Leo, 1996). Per l'Arcipelago Toscano sono note due sottospecie: $C$. strigosus oglasensis Gardini, 1976, descritto di Montecristo e successivamente citato per l'Isolotto La Scola (Lo Cascio et al., 2000) e C. strigosus strigosus, noto solo dell'Elba (Gardini, 1976) e qui segnalato per la prima volta per il Giglio e l'isolotto di Palmaiola.

Uloma (Uloma) culinaris (Linnaeus, 1758)

RePERTI: Isola d'Elba, Portoferraio, San Martino, str. Romana, 5.I.2020, LF, 1 ex (CLF). 
Note. Specie ad ampia distribuzione europea (Löbl et al., 2008), segnalata genericamente di tutta Italia da Luigioni (1929) e Porta (1934). Prima segnalazione (sia della specie che della tribù Ulomini) per l'Elba e per l'Arcipelago Toscano.

Crypticus (Crypticus) gibbulus (Quensel, 1806)

RePERTI: Isola di Montecristo, Cala Maestra, 78.VI.205, LF, 6 ex (CLF, CPL).

NotE. Specie ampiamente diffusa nella regione mediterranea (Löbl et al., 2008), frequente in Sardegna e Sicilia, già nota per l'Arcipelago Toscano solo del Giglio (Gardini, 1976); per l'Italia peninsulare la specie è citata di due sole stazioni: Santa Marinella nel Lazio (Gridelli, 1949) e Le Tofane (presso Alberese) in Toscana (Marcuzzi, 1997). Prima segnalazione per Montecristo, benché a questa stessa specie sia evidentemente da riferire la segnalazione di un resto da parte di Poggi (1976, sub “Crypticus sp.") per la stessa località.

Diaperis boleti (Linnaeus, 1758)

RePERTI: Isola d'Elba, Campo nell'Elba, M. Perone, 400 m, 19.VI.2011, LF, 3 ex (CLF); idem, frazione San Piero, strada per M. Perone, 400 m, 19.VII.2011, sotto corteccia di Castanea sativa, LF, 6 ex (CPL); idem, M. Capanne, cabinovia, 29.VIII.2012, LF, 1 ex (CLF).

NotE. Specie ad ampia distribuzione sibirico-europea, presente anche nel Maghreb (Löbl et al., 2008) e segnalata genericamente di tutta Italia da Luigioni (1929) e Porta (1934). Prima segnalazione per l'Elba e per l'Arcipelago Toscano.

Pentaphyllus chrysomeloides (Rossi, 1792)

REPERTI: Isola d'Elba, Portoferraio, Acquabona, Monte Fabrello, 7.IX.2012, LF, 5 ex (CLF); idem, 1.X.2012, LF, 2 ex (CLF).

Note. Specie diffusa in modo discontinuo in Europa e nell'Asia centro-occidentale (Löbl et al., 2008), nota di varie regioni italiane, prevalentemente del centrosud (Aliquò et al., 2007). Prima segnalazione per l'Elba e per l'Arcipelago Toscano.

\section{Corticeus (Corticeus) pini (Panzer, 1799)}

REPERTI: Isola d'Elba, Campo nell'Elba, frazione San Piero, Piana Canale, 13.III.2016, LF, 3 ex (CLF).

Note. Specie a distribuzione ampia ma discontinua nella regione paleartica (Löbl et al., 2008), presente nella maggior parte delle regioni italiane (Leo, 1991;
Aliquò et al., 2007). Prima segnalazione (sia della specie che della tribù Hypophlaeini) per l'Elba e per l'Arcipelago Toscano.

Phaleria (Phaleria) acuminata acuminata Küster, 1852

RePERTI: Isola di Pianosa, 20.V.2000, N. Baccetti leg., 49 ex (CPL); idem, dintorni paese, 6.V.2014, LF, 4 ex (CLF, CPL).

Note. Specie ad ampia distribuzione mediterranea (Löbl et al., 2008), con alcune sottospecie di dubbio valore tassonomico, presente lungo le coste sabbiose dell'Italia meridionale e centrale, a nord fino alle Marche e alla Toscana (Papi \& Ceccolini, 2014); la presenza di questa specie lungo le coste delle regioni più settentrionali, riportate in mappa da Aliquò et al. (2007), sembra sia da escludere. Prima segnalazione per Pianosa e per l'Arcipelago Toscano.

Phaleria (Phaleria) bimaculata bimaculata (Linnaeus, 1767)

REPERTI: Isola di Montecristo, Cala Maestra, 9.VI.2015, LF, 10 ex (CLF, CPL); idem, idem, 2.X.3016, LF, 4 ex (CLF, CPL).

Note. Specie ad ampia distribuzione mediterranea (Löbl et al., 2008), divisa in varie sottospecie di dubbia validità; presente lungo le coste di quasi tutte le regioni italiane (Aliquò et al., 2007). Già citata per l'Arcipelago Toscano di Elba, Giglio, Capraia (Gardini, 1976) e Pianosa (Lo Cascio et al., 2000). Prima segnalazione per Montecristo.

Scaphidema metallica (Fabricius, 1792) REPERTI: Isola d'Elba, Campo nell'Elba, Castagnone, 400 m, 4.V.2014, LF, 1 ex (CLF); idem, Portoferraio, Albereto, Monte Bello, 75 m, 1.IX.2014, LF, 1 ex (CLF).

Note. In accordo con Löbl \& Smetana (2010) il taxon Scaphidema Redtenbacher, 1849 è considerato di genere femminile. Specie sibirico-europea, nota dell'Italia settentrionale, Toscana, Marche, Lazio, Puglia, Basilicata e Calabria (Giovagnoli et al., 2012). Prima segnalazione (sia della specie che della tribù Scaphidemini) per l'Elba e per l'Arcipelago Toscano.

\section{CONCLUSIONI}

Per l'Arcipelago Toscano erano fino ad oggi note 53 specie (cfr. Gardini, 1976, 1979; Leo, 1998, 2008; Lo Cascio et al., 2000). Al fine dell'esame dei popolamenti naturali di arcipelago e singole isole, ab- 
biamo comunque escluso le specie che riteniamo di sicura introduzione antropica; in particolare Blaps lethifera Marsham, 1802, che in Italia è esclusivamente sinantropa, e le diverse specie ad attuale distribuzione cosmopolita, legate alle derrate alimentari: Alphitobius diaperinus, Palorus subdepressus (Wollaston, 1864), Tenebrio molitor Linnaeus, 1758, T. obscurus Fabricius, 1792, Tribolium castaneum (Herbst, 1797), $T$, confusum Jacquelin du Val, 1868, Gnatocerus cornutus (Fabricius, 1798) (cfr. anche Fattorini, 2009). Quindi, con l'esclusione dei taxa di origine antropica di cui sopra, abbiamo 45 specie già segnalate che, con l'aggiunta delle 9 nuove per l'arcipelago (Dichillus corsicus, Eledona agricola, Palorus depressus, Uloma culinaris, Diaperis boleti, Pentaphyllus chrysomeloides, Corticeus pini, Phaleria acuminata acuminata, Scaphidema metallica), porta il totale a 54; l'incremento di specie di Tenebrionidae note dell'Arcipelago Toscano è quindi del $20.0 \%$, assolutamente notevole per un popolamento faunistico che si riteneva ben conosciuto almeno per le isole maggiori (cfr. Fattorini, 2009).

Per quanto riguarda le singole isole, è rimarchevole che le specie nuove dell'Elba, che pure si riteneva tra le isole meglio esplorate (cfr. Gardini, 1976), siano ben sette (con un incremento del 21,2\%: da 33 a 40 specie) e tutte segnalate per la prima volta per la fauna dell'intero arcipelago (come già detto, non viene qui considerata la specie cosmopolita Alphitobius diaperinus). Pianosa è stata oggetto di ricerche approfondite in tempi relativamente recenti (cfr. Lo Cascio et al., 2000), tuttavia ci è stato possibile riportare tre nuove specie per l'isola (con un incremento del 15,8\%: da 19 a 22 specie). I Tenebrionidi di Montecristo, isola evidentemente fino ad oggi poco esplorata, passano da 9 a 12 specie (incremento del 33,3\%). Gli isolotti minori sono quelli nei quali le conoscenze sono più carenti; emblematico il caso di Cerboli, di cui si conoscevano solo tre specie, passate a cinque (incremento del 66,7\%) pur con solo qualche ora di ricerca in loco, effettuata tra l'altro in un periodo poco propizio (fine agosto). Riportiamo infine il caso di Palmaiola, per la quale vengono qui citati i primi reperti di Tenebrionidae, relativi a tre specie già note della vicina Elba.

\section{RINGRAZIAMENTI}

Gli autori desiderano ringraziare in particolar modo il PNAT (Parco Nazionale dell'Arcipelago Toscano) che ha autorizzato e tuttora continua a favorire le ricerche sull'entomofauna delle isole dell'arcipelago Toscano, i Carabinieri Forestali per l'aiuto logistico nel corso dei vari accessi e durante la permanenza sull'Isola di Montecristo e l'Amministrazione Penitenziaria per la disponibilità dimostrata nel fornire supporto logistico durante le ricerche sulle Isole di Gorgona e Pianosa. Ringraziamo inoltre la W.B.A. (World Biodiversity association onlus) di Verona per il prezioso supporto scientifico, organizzativo e logistico fornito nel corso delle ricerche.

\section{BIBLIOGRAFIA}

Aliquò V., Leo P., 1996 - I coleotteri Tenebrionidi delle Madonie (Sicilia) (Coleoptera Tenebrionidae). Il Naturalista siciliano, Palermo, S.4, 20(3-4): 281-304.

Aliquò V., Rastelli M., Rastelli S., Soldati F., 2007 - Coleotteri Tenebrionidi d'Italia - Darkling Beetles of Italy. Piccole Faune, Associazione Naturalistica Piemontese, CD-ROM.

FAtTorini S., 2007. The Tenebrionid beetles of Mt Vesuvius: species assemblages and biogeographic kinetics on an active volcano (Coleoptera: Tenebrionidae) (pp. 221-247). In: NARDi G., Vomero V. (eds.), Artropodi del Parco Nazionale del Vesuvio: ricerche preliminari. Conservazione Habitat Invertebrati, 4. Cierre Edizioni, Verona, 496 pp.

FATTORINI S., 2009 - Faunal patterns in tenebrionids (Coleoptera: Tenebrionidae) on the Tuscan Islands: The dominance of paleogeography over Recent geography. European Journal of Entomology, 106: 415-423.

FATTORINI S., 2013 - I Coleotteri Tenebrionidi di Roma (Coleoptera, Tenebrionidae). Fragmenta entomologica, 45(1-2): 87-142.

FAtToRini S., Leo P., 2000 - Darkling beetles from Mediterranean minor islands: new records and biogeographical notes (Coleoptera Tenebrionidae). Bollettino della Società entomologica italiana, 132: 205-217.

GARDini G., 1976 - Materiali per lo studio dei Tenebrionidi dell'Arcipelago Toscano (Col. Heteromera). Lavori della Società Italiana di Biogeografia, (n.s.), 5 (1974): 637-723.

GARDINI G., 1979 - Nuovi dati sui Tenebrionidi (Col.) dell'Arcipelago Toscano. Bollettino del Museo civico di storia naturale di Verona, 6: 73-77. 
Giovagnoli G., Strocchi A., Paglialunga M., 2012 - Coleotteri della Regione Marche. Primo contributo alla conoscenza della coleotterofauna della Regione Marche (Insecta Coleoptera Carabidae, Buprestidae, Meloidae, Tenebrionidae, Lucanidae, Bolboceratidae, Melolonthidae, Cetoniidae, Cerambycidae). Quaderni di Studi e notizie di Storia naturale della Romagna, 36: 159-184.

Gridelli E., 1949 - Gen. Crypticus (pp. 271-272). In: Porta A. (ed.), Fauna Coleopterorum Italica. Supplementum II. Stabilimento Tipografico S.A.G. Gandolfi, San Remo, 387 pp.

Iwan D., Ferrer J., Raś. M., 2010 - Catalogue of the World Gonocephalum Solier, 1834 (Coleoptera, Tenebrionidae, Opatrini). Part 1. List of the species and subspecies. Annales Zoologici, 60: 245-304.

Leo P., 1991 - Hypophloeus (Paraphloeus) pini (Panzer, 1799) (Coleoptera Tenebrionidae). Segnal. faun. ital. n. 187. Bollettino della Società entomologica italiana, 123(1): 73-74.

LEO P., 1998 - Nuovi dati sui tenebrionidi delle isole toscane e descrizione di Asida (s. str.) gestroi Leoni lanzai n. ssp. (Coleoptera, Heteromera). Atti del Museo di storia naturale della Maremma, 17: 73-77.

Leo P., 2008 - Osservazioni su Dichillus corsicus e descrizione di tre nuove specie del Mediterraneo occidentale (Coleopera, Tenebrionidae). Annali del Museo civico di storia naturale "G. Doria”, 99: 603-627.

LEO P., 2015 - Nuovi dati sulla distribuzione di Dichillus (Dichillus) minutus (Solier, 1838) (Coleoptera: Tenebrionidae). Onychium, 11: 143-145.

Löbl I., Ando K., Bouchard P., Iwan D., Lillig M., Masumoto K., Merkl O., Nabozhenko M., Novák V., Petterson R., Schawaller W., Soldati F., 2008 - Family Tenebrionidae Latreille, 1802 (pp. 105-325). In: Löbl I., Smetana A. (eds), Catalogue of Palaearctic Coleoptera. Volume 5. Tenebrionoidea. Apollo Books, Stenstrup, 670 pp.

LöBl I., Smetana A. (eds), 2010 - Errata for vol. 5, (pp. 29-36). In: Catalogue of Palearctic Coleoptera vol. 6. Apollo Books, Stenstrup, $924 \mathrm{pp}$.

Lo Cascio P., 2001 - Tenebrionidae, pp. 219-228 - In: SForzi A., BARTolozzi L. (eds), Libro Rosso degli insetti della Toscana. Arsia, Firenze, 376 pp.

Lo Cascio P., Bartolozzi L., Cecchi L., Dapporto L., Sforzi A., 2000 - Contributi alla conoscenza dell'artropodofauna dell'Isola di Pianosa (Arcipelago Toscano). 3. Coleoptera Tenebrionidae. Bollettino della Società entomologica italiana, 132(2): 157-174.

Luigioni P., 1929 - I Coleotteri d'Italia. Catalogo sinonimico-topografico-bibliografico. Memorie della pontificia accademia delle Scienze "I nuovi Lincei", 2(13): 1-1160.

PApi R., Ceccolini F., 2014 - Nuovi dati corologici per alcune specie italiane di Tenebrionidi (Coleoptera: Tenebrionidae). Onychium, 10 (2013): 127-132.

Poggi R., 1976 - Materiali per un elenco dei Coleotteri dell'isola di Montecristo (Arcipelago toscano) con descrizione di un nuovo Stafilinide (Leptotyphlus oglasensis n.sp.) (Studi sulla Riserva Naturale dell'Isola di Montecristo. XV). Lavori della Società italiana di Biogeografia, (n.s.), 5 (1974): 609- 635.

PorTA A., 1934 - Fauna Coleopterorum Italica, Heteromera-Phytophaga. Vol.IV. Heteromera Phytophaga. Stabilimento Tipografico Piacentino, Piacenza, 415 pp. 
